\title{
Adoção de tecnologia em organizações públicas brasileiras à luz do modelo UTAUT: uma revisão sistemática da literatura.
}

\author{
Andrea Leal Barros de Melo Salles - lealsuri@gmail.com ${ }^{1}$ \\ Natalia Melo da Silva - natalia.melos@univasf.edu.br ${ }^{1}$ \\ Platini Gomes Fonseca-platini.fonseca@univasf.edu.br ${ }^{1}$ \\ Ernani Marques dos Santos -ernanims@gmail.com²
}

Resumo - O objetivo deste artigo foi, através de uma revisão sistemática, analisar a literatura que utiliza o modelo UTAUT para compreender a adoção de tecnologia em organizações públicas, a partir dos seguintes aspectos de análise: objetivo dos artigos encontrados; contextos de aplicação; metodologia utilizada; construtos adaptados, limitações da pesquisa e indicações de pesquisas futuras. Os resultados apontaram que o contexto público tem sido preterido pelos pesquisadores, quando comparado às pesquisas que estudam a adoção de tecnologia em organizações privadas. Conclui-se que a maioria dos artigos selecionados realizaram adaptações no modelo UTAUT quando utilizados em organizações públicas. Contudo, os resultados não permitem afirmar as razões para tais alterações, se estão relacionadas à tecnologia empregada, ou às características do serviço público. Como contribuição complementar, o artigo buscou construir um protocolo de revisão sistemática abrangente.

Palavras-chaves: Aceitação e uso de tecnologia; Modelo UTAUT; Serviço público.

\section{Technology adoption in Brazilian public organizations in the light of the UTAUT model: a systematic literature review.}

\begin{abstract}
The objective of this study was, through a systematic review, to analyze the literature that uses the UTAUT model to understand technology adoption in public organizations based on the following aspects of analysis: the purpose of the articles found; contexts of applicability; methodology employed; constructs adapted; research limitations; and future research indications. The results suggest that the public context has been disregarded by researchers when compared to studies that assess technology adoption in private organizations. It is concluded that most of the articles selected made adaptations in the UTAUT model when used in public organizations. However, the results do not allow to state the reasons for such modifications, whether they are related to the technology employed or the characteristics of public service. As a supplementary contribution, the article aimed to construct a comprehensive systematic review protocol.
\end{abstract}

Keywords: Acceptance and use of technology; UTAUT model; Public service.

Data da Submissão: 27/01/2021

Data de aceitação: 11/03/2021 


\section{Introdução}

Apesar da quantidade de pesquisas amplamente utilizadas em diferentes setores do setor privado, ainda existe a necessidade de se avançar nas publicações sobre o tema no contexto de órgãos públicos (GUIMARÃES; MEDEIROS, 2005); (ROZTOCKI; STRZELCZYK, 2020). Essa afirmação tem se confirmado em revisões sistemáticas da literatura que buscam comparar a ênfase que os pesquisadores dão às organizações privadas, preterindo investigações que levem em conta características e peculiaridades dos órgãos públicos (MIRANDA, 2018); (FONSECA et al., 2017a, 2017b; 2018; 2019).

Por exemplo, Sena e Guarnieri (2015), ao abordarem pesquisas a respeito de sistemas Enterprise Resource Planning (ERP), confirmam que embora exista um arcabouço teórico já consolidado acerca dos sistemas na área privada, ainda se carece de pesquisas que tratem da área pública - uma vez que somente nos últimos anos, organizações governamentais nacionais passaram a implementar esse tipo de sistema.

Além da possível lacuna contextual que se apresenta, faz-se necessário conhecer como um dos modelos de aceitação tecnológica tem sido utilizado para o entendimento da adoção de tecnologia nessas organizações, sobremaneira o comportamento do usuário em relação à aceitação da tecnologia da informação - a exemplo da Teoria Unificada de Aceitação e Uso de Tecnologia (VENKATESH et al., 2003); (ANDWIKA, 2020); (BRITO, 2019); (SOLIMAN, 2019);

O modelo UTAUT, desenvolvido por Venkatesh, Morris, Davis e Davis (2003), aborda e integra elementos de diversas teorias que visam compreender a adoção tecnológica (GONZALES et al., 2017). Na revisão sistemática da literatura realizada por Gonzales et al., (2017), os autores concluíram, dentre outros achados, que a aplicação do modelo tem acontecido em uma variedade de ramos empresariais - com uma leve concentração em setores educacionais e comerciais. Entretanto, a pesquisa não distingue se o contexto é público ou privado.

Essa problematização exige a elaboração da seguinte questão de pesquisa: como a literatura tem utilizado o modelo UTAUT para compreender a adoção de tecnologia em organizações públicas? Assim, o artigo tem o como objetivo analisar sistematicamente a literatura que utiliza o modelo UTAUT para compreender a adoção de tecnologia em organizações públicas, a partir dos seguintes aspectos de análise: objetivo dos artigos encontrados; contextos de aplicação; metodologia utilizada; construtos adaptados, limitações da pesquisa e indicações de pesquisas futuras. Como contribuição complementar, o artigo buscou construir um protocolo de revisão sistemática abrangente.

Esse artigo está organizado em cinco seções, além das indicações das referências utilizadas: a introdução, o debate teórico, a explicação metodológica, a apresentação e análise dos resultados e considerações finais.

\section{Discussão Conceitual}

Faz-se necessário debater a respeito dos fundamentos e conceitos que embasaram análise desta pesquisa. Por essa razão, as próximas seções foram reservadas à construção das ideias em torno da Adoção de tecnologia no âmbito do Serviço Público, assim como, a exposição do Modelo UTAUT. 


\subsection{Adoção de Tecnologia no Serviço Público}

Os anos 1990 foi uma década de grandes transformações globais. Diversos países, em prol da competitividade, foram impulsionados a se adaptar às novas demandas do capitalismo, frente à presença da globalização; assim como também, das latentes transformações político-sociais e do uso de novas tecnologias.

De acordo com Bresser-Pereira (1998) a onda neoconservadora e as reformas econômicas orientadas para o mercado foram responsáveis pela crise eclodida na década anterior. Ao passo que, nos anos 1990, constatou-se a inviabilidade da proposta conservadora de Estado mínimo, o que significou uma condição necessária às reformas de reconstrução do Estado. Não apenas tarefas clássicas de garantia da propriedade e dos contratos deveriam ser realizadas, mas também, o papel de garantidor dos direitos sociais e de promotor da competitividade do seu respectivo país.

O fato é que tais mudanças exigiram da Administração Pública, e consequentemente às organizações públicas, uma roupagem compatível com que se propusera a reforma em curso, em que o objetivo era a substituição de uma Administração Pública Burocrática por uma Administração Pública Gerencial.

Consequentemente, estas demandas impuseram novas exigências às instituições públicas. Amplia-se a necessidade e a possibilidade de formar pessoas capazes de lidar com o avanço da ciência e da tecnologia, prepará-los para o mundo contemporâneo e dele participarem de forma dinâmica. Com isso, também surge à necessidade de adequação a novas rotinas de trabalho e do uso de estruturas descentralizadas e flexíveis. O objetivo era melhorar o ambiente institucional e atender a sociedade com serviços de qualidade e com gestores éticos e responsáveis para promover modelos de gestão mais profissionais e eficientes. (OLIVEIRA, 2017).

No Brasil, tal dinâmica se deu a partir do Plano Diretor elaborado no primeiro mandato do então presidente Fernando Henrique Cardoso, sob a coordenação do Ministro da Administração Federal e Reforma do Estado Luiz Carlos Bresser Pereira. Deu-se início a uma nova maneira de se pensar a "Coisa Pública".

Nesse contexto de mudanças, ganham força políticas públicas com foco na inovação, como o governo eletrônico ou e-gov. No Brasil, é possível observar em dados coletados no sítio eletrônico "governo digital", vinculado ao governo federal, que desde o ano 2000, o governo brasileiro tem buscado evoluir seus processos e a prestação de serviços públicos com o auxílio das Tecnologias da Informação e Comunicação (TIC). Destaca-se que o Programa de Governo Eletrônico iniciou no Brasil uma série de adaptações, inovações e desafios para a realização da melhoria da qualidade do serviço público.

Para isso, diversas políticas e iniciativas foram realizadas até 2016, mas com a publicação da Estratégia de Governança Digital (EGD), foi implantado um novo paradigma de gestão pública e das relações entre o Estado brasileiro e a Sociedade. Ações voltadas à desburocratização, modernização do Estado, simplificação de processos, melhoria no acesso à informação pública, transparência, melhoria nos atendimentos e racionalização de gastos públicos são alguns avanços que a política de governança eletrônica e digital proporcionaram. (GOVERNO FEDERAL, 2020) 
Além disso, Medeiros e Guimarães (2003) levantam outros aspectos que podem ter incentivado o surgimento dessa inovação no serviço público: a necessidade das administrações aumentarem sua arrecadação e melhorarem seus processos internos e as pressões da sociedade para que o governo otimizasse seus gastos e atuasse, cada vez mais, com transparência, qualidade na oferta de serviços aos cidadãos e às organizações em geral; e as possibilidades de otimização de processos de gestão governamental.

Vale destaque, que muitas das tendências tecnológicas adotadas no setor público originaram-se no setor privado (RORATTO; DIAS, 2012). As tecnologias associadas com e-business, por exemplo, foram adotadas nas organizações públicas e trouxeram mudanças ao setor público semelhante às observadas no setor privado. Governos em todos os níveis estão usando a Internet para fornecer informações e serviços aos cidadãos, funcionários e empresas com as quais trabalham. (LAUDON; LAUDON, 2014).

Outra consequência a essas mudanças foram os estudos sobre a Aceitação e Uso de Tecnologia que ganharam espaço na literatura científica. Isso porque consoante Venkatesth et al., (2003), os usuários e as organizações reagem de forma diferente entre si em relação às novas tecnologias, tanto na adoção quanto na resistência. Com isto, pesquisas foram realizadas buscando compreender a ação do indivíduo sobre adotar ou não uma tecnologia e quais os fatores influenciavam essa decisão (DAVIS, 1989).

A não atenção a essa constatação pode implicar, por exemplo, em prejuízos diante dos altos investimentos direcionados ao desenvolvimento de TI, em busca do desenvolvimento na produtividade das organizações, quando desconectados da aceitação do usuário final. Pois para que ocorram bons resultados, as inovações precisam ser aceitas e efetivamente utilizadas nas organizações.

Dado exposto acima é possível justificar o emprego no contexto predominante privado dos estudos sobre a temática. Como também, reforça a importância do prosseguimento de investigações em outro cenário não tão explorado - foco de interesse deste estudo - o setor público. Visto que apesar da aceitação e uso de novas tecnologias possuírem uma das literaturas mais maduras na área de Sistema de Informação, ao mesmo tempo, de acordo com Venkatesh et al (2003), há várias fragmentações capazes de explicar a adoção da tecnologia individual e organizacional.

Diante dessa complexidade, diversos modelos e teorias foram desenvolvidas por diferentes pesquisadores de adoção de tecnologia. Um dos modelos mais consagrados é a Teoria Unificada de Aceitação e Uso da Tecnologia ou "Unified Theory of Acceptance and Use of Technology" (UTAUT), que foi formulado por Venkatesh e outros. Modelo selecionado para o desenvolvimento do presente estudo e que será detalhado a seguir.

\subsection{Aceitação do Uso de Tecnologia e o Modelo UTAUT}

Conforme já mencionado acima, em virtude do forte investimento e consequente implementação de diversas tecnologias em organizações pelo mundo, surgiu o interesse no desenvolvimento de pesquisas que pudessem explicar a relação entre aceitação e uso de tecnologia e o alcance dos resultados esperados. (VENKATESH et al., 2003) 
Diante disso, o modelo de aceitação e uso de tecnologia (UTAUT) foi resultado da pesquisa desenvolvida por Venkatesh e outros, no ano de 2003, na revista MIS Quartely, que incorporou elementos de outros oito modelos de interesse à aceitação de tecnologia: Teoria da Ação Racional (TRA), proposta por Fishbein e Ajzen (1975); o Modelo de Aceitação da Tecnologia (TAM), de Davis (1989); o Modelo Motivacional (MM), de Vallerand (1997); a Teoria do Comportamento Planejado (TPB), de Ajzen (1991); o Modelo Combinado TAM-TPB, proposto por Taylor e Tood (1995); o Modelo de Utilização do PC (MPCU), de Thompson, Higgins (1991); a Teoria da Difusão da Inovação, de Rogers (1995), aplicada em SI por Moore e Benbasat (1996); Teoria Social Cognitiva, de Bandura (1986), ampliada para o contexto de uso de computadores por Compeau e Higgins (1995). (VENKATESH, 2003)

O objetivo do UTAUT é servir como ferramenta mais completa à gestão de instituições que necessitem avaliar a probabilidade de sucesso de novas tecnologias, além de colaborar na compreensão de motivadores de aceitação, intrísecos e extrísecos, o que permite uma projeção proativa em ações interventoras (como treinamento, marketing, etc.), que podem ser direcionadas a populações de usuários menos inclinados a adotar e usar novos sistemas. (VENKATESH, 2003)

A Teoria Unificada de Aceitação e Uso da Tecnologia apresenta quatro construtos que desempenham um papel determinante na aceitação e comportamento de uso do usuário, que são: "Expectativa de Desempenho"; "Expectativa de Esforço"; "Influência Social" e "Condições Facilitadoras". Os resultados surgem de acordo com a associação destes construtos com seus moderadores: "Gênero"; "Idade"; "Experiência" e "Voluntariedade de uso", aplicados conforme a Figura 1. (Venkatesh et al., 2003).

Figura 1- Constructos essenciais do modelo UTAUT.

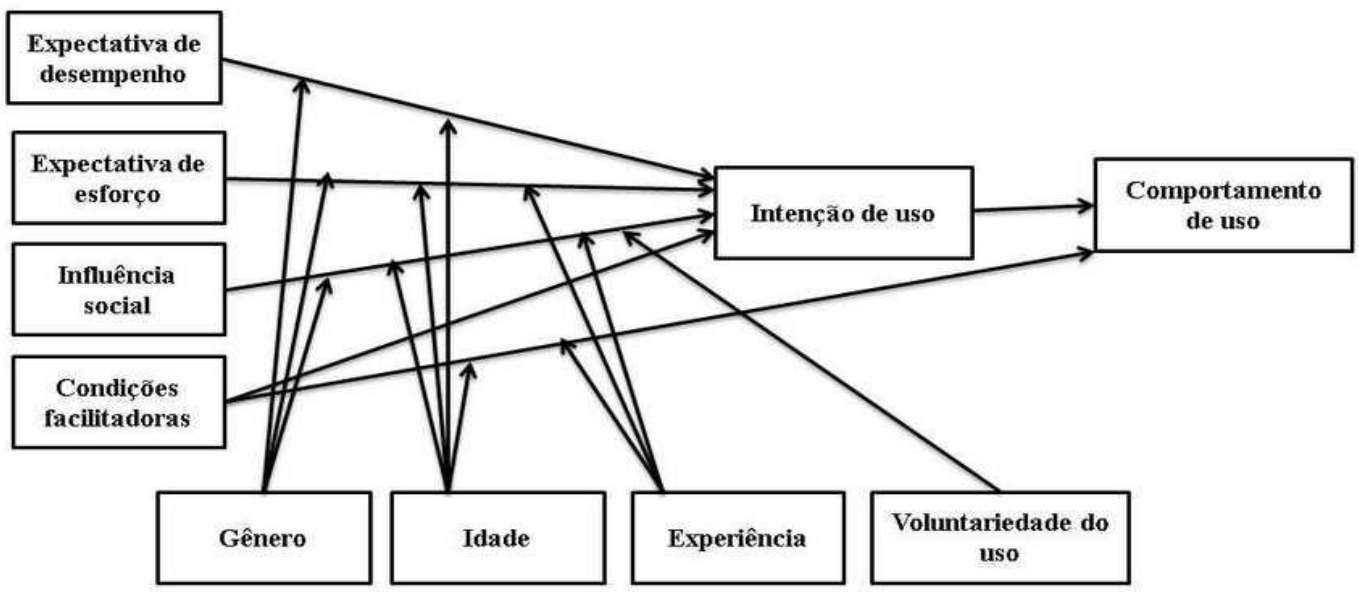

Fonte: Adaptado de Venkatesh et al. (2003).

Ademais, as definições desses construtos segundo Venkatesh et al. (2003) são: a) expectativa de desempenho é definida como o grau em que um indivíduo acredita que usar o sistema vai ajudá-lo a obter ganhos em suas atribuições profissionais; b) expectativa de esforço é definida como o grau de facilidade associados ao uso da tecnologia; c) influência social é definida como o grau em que um indivíduo percebe que outros acreditam que ele deve usar o novo sistema; e d) condições facilitadoras são definidas como o grau em que um indivíduo acredita que a organização dispõe de infraestrutura técnica para apoiar o uso da tecnologia. 
Vale destacar ainda, que a escolha do UTAUT para o desenvolvimento desta pesquisa ocorreu em virtude do modelo em primeiro lugar oportunizar uma análise com viés tanto individual quanto organizacional (VENKATESH, 2003); (MOSWEU, 2015);(GONZALES, 2017), em segundo lugar, ele explicou que $70 \%$ da variância da intenção de comportamento e $48 \%$ para comportamento de uso, sendo considerado eficaz para predizer a aceitação e o uso de tecnologia no contexto das organizações (GONZALES, 2017), enquanto que - na intenção de uso - obtido com a aplicação dos outros oito modelos, obtêm-se percentuais entre $17 \%$ a $53 \%$. (VENKATESH, 2003);(SILVA, 2017).

Além disso, a observância de uma constante aplicação do modelo em pesquisas acadêmicas, nos últimos anos, tanto internacionalmente como no Brasil, o que corrobora com a ideia de ser um modelo consolidado cientificamente.

\section{Explicação Metodológica}

Acham-se descritos a seguir os steps do percurso metodológico.

\subsection{Revisão Sistemática da literatura}

Como abordagem metodológica, optou-se por utilizar a revisão sistemática da literatura. De acordo com Kitchenham et al. (2007), a revisão sistemática da literatura é um meio de avaliar e interpretar todas as pesquisas disponíveis relevantes para uma determinada questão de pesquisa, área de tópico ou fenômeno de interesse.

Esse método visa apresentar uma avaliação justa de um tópico de pesquisa, usando procedimentos rigorosos e explícitos, de maneira que os resultados encontrados sejam completos e eficientes (KITCHENHAM et. al, 2007); (CASSUNDÉ, BARBOSA, MENDONÇA, 2018). Para tanto, Cassundé, Barbosa e Mendonça (2018) recomendam a realização de três etapas: 1) Definir o objetivo da Revisão; 2) Identificar a literatura; e 3) Selecionar os possíveis estudos a serem incluídos.

$\mathrm{Na}$ presente pesquisa, foram executadas duas revisões sistemáticas da literatura. $\mathrm{Na}$ primeira, buscou-se fazer um levantamento sobre os protocolos utilizados em outras revisões sistemáticas realizadas na área de gestão de tecnologia, de modo a construir um protocolo de revisão sistemática abrangente. Após a elaboração do protocolo abrangente, o mesmo foi aplicado para balizar a segunda revisão sistemática, que teve como escopo responder à questão de pesquisa proposta, qual seja, analisar a literatura que utiliza o modelo UTAUT para compreender a adoção de tecnologia em organizações públicas.

\subsection{Construção do protocolo abrangente}

Para a construção do protocolo abrangente, realizou-se uma busca por artigos científicos que usaram como metodologia a revisão sistemática e que tenham sido publicados nas edições especiais (Anais do Simpósio Brasileiro de Tecnologia da Informação) da Revista Gestão.Org dos últimos 05 (cinco) anos. A busca foi realizada de forma manual, no endereço eletrônico da Revista (https://periodicos.ufpe.br/revistas/gestaoorg), a partir da leitura do título e do resumo de todos os artigos publicados nas edições especiais no interregno de tempo definido, tendo como resultado 09 (nove) artigos. 
Após a seleção dos 09 (nove) artigos que atendiam aos requisitos acima descritos, foi feita uma leitura atenta do capítulo de metodologia de cada um deles, observando os critérios utilizados pelos autores no protocolo de revisão. O objetivo era fazer um levantamento de todos os critérios eleitos nos protocolos das revisões sistemáticas selecionadas, para construir um novo protocolo que contemple todos esses critérios, denominado pelos pesquisadores como "protocolo abrangente".

Os critérios identificados foram: definição do banco de dados para realizar a pesquisa; corte temporal; previsão de palavras-chave em português e/ou em língua estrangeira; definição da forma de busca (se foi utilizado motor de busca ou busca manual, por exemplo), critérios de inclusão; critérios de exclusão; avaliação de qualidade dos artigos; se foi especificada alguma forma de extração de dados, e, por fim, se foi definida como se daria a análise dos artigos. A tabela abaixo ilustra os 09 (nove) artigos selecionados, bem como os critérios identificados nos protocolos de revisão de cada um deles. A letra " $x$ " foi colocada nos critérios localizados em cada artigo. Por outro lado, os espaços em branco indicam que determinado critério não foi localizado naquele artigo.

Quadro 1 - Elementos definidores do protocolo de busca.

\begin{tabular}{|c|c|c|c|c|c|c|c|c|c|c|}
\hline Artigos & $\begin{array}{c}\text { Banco } \\
\text { de } \\
\text { dados }\end{array}$ & $\begin{array}{l}\text { Corte } \\
\text { temporal }\end{array}$ & $\begin{array}{l}\text { Palavras- } \\
\text { chave em } \\
\text { português }\end{array}$ & $\begin{array}{l}\text { Keyword } \\
\text { inglês }\end{array}$ & $\begin{array}{l}\text { Forma } \\
\text { de } \\
\text { Busca }\end{array}$ & $\begin{array}{l}\text { Critério } \\
\text { de } \\
\text { inclusão }\end{array}$ & $\begin{array}{l}\text { Critério } \\
\text { de } \\
\text { exclusão }\end{array}$ & $\begin{array}{c}\text { Avalia } \\
\text { qualidade }\end{array}$ & $\begin{array}{l}\text { Extrair } \\
\text { dados }\end{array}$ & $\begin{array}{l}\text { Análise } \\
\text { do artigo }\end{array}$ \\
\hline $\begin{array}{l}\text { SANTOS; DEDA; } \\
\text { OLIVEIRA, 2015. }\end{array}$ & $\mathrm{x}$ & $\mathrm{x}$ & & $\mathrm{x}$ & $\mathrm{x}$ & $\mathrm{x}$ & $\mathrm{x}$ & & & $\mathrm{x}$ \\
\hline $\begin{array}{l}\text { SILVA; GARCIA; } \\
\text { NASCIMENTO, } 2016\end{array}$ & $\mathrm{x}$ & & & $\mathrm{x}$ & $\mathrm{x}$ & & $\mathrm{x}$ & & $\mathrm{x}$ & $\mathrm{x}$ \\
\hline $\begin{array}{l}\text { MAGNAGNAGNO; } \\
\text { LUCIANO; } \\
\text { WIEDENHOFT, } 2017\end{array}$ & $\mathrm{x}$ & $\mathrm{x}$ & $\mathrm{x}$ & $\mathrm{x}$ & & $\mathrm{x}$ & $\mathrm{x}$ & $\mathrm{x}$ & $\mathrm{x}$ & $\mathrm{x}$ \\
\hline $\begin{array}{l}\text { FERREIRA; PINTO; } \\
\text { SANTOS, } 2017\end{array}$ & $\mathrm{x}$ & $\mathrm{x}$ & & $\mathrm{x}$ & $\mathrm{x}$ & $\mathrm{x}$ & $\mathrm{x}$ & $\mathrm{x}$ & $\mathrm{x}$ & $\mathrm{x}$ \\
\hline $\begin{array}{l}\text { MIRANDA; } \\
\text { VERÍSSIMO; CEOLIN, } \\
2017\end{array}$ & $\mathrm{x}$ & $\mathrm{X}$ & & & & $\mathrm{x}$ & & & & \\
\hline ARAUJO et. al, 2018 & $\mathrm{x}$ & $\mathrm{x}$ & & $\mathrm{x}$ & $\mathrm{x}$ & $\mathrm{x}$ & $\mathrm{x}$ & $\mathrm{x}$ & $\mathrm{x}$ & \\
\hline $\begin{array}{l}\text { LOPES; LUCIANO; } \\
\text { MACADAR, } 2018 .\end{array}$ & $\mathrm{x}$ & & $\mathrm{x}$ & $\mathrm{x}$ & & & & & $\mathrm{x}$ & $\mathrm{x}$ \\
\hline SILVA; BURÉGIO, 2018. & $\mathrm{x}$ & $\mathrm{x}$ & $\mathrm{x}$ & & & & & & & \\
\hline
\end{tabular}




\begin{tabular}{|l|c|c|c|c|c|c|c|c|c|}
\hline NEVES $e$ t. al, 2019 & $\mathrm{X}$ & $\mathrm{X}$ & $\mathrm{X}$ & $\mathrm{X}$ & & $\mathrm{X}$ & & $\mathrm{X}$ & \\
\hline
\end{tabular}

Fonte: Elaborado pelos autores.

Nesse sentido, o protocolo abrangente montado pelos autores contemplou todos os critérios identificados nos artigos de revisão sistemática selecionados, conforme demonstração na tabela acima, quais sejam: banco de dados; corte temporal; palavras-chave em português; palavras-chave em língua estrangeira; forma de busca; critérios de inclusão; critérios de exclusão; avaliação de qualidade dos artigos; extração de dados, e, por fim, a análise dos artigos.

\section{3 - Planejamento da pesquisa.}

Conforme já mencionado, a segunda revisão sistemática foi feita para buscar artigos científicos que estudassem a aplicação do modelo UTAUT para compreender a adoção de tecnologia em organizações públicas, utilizando o protocolo abrangente construído conforme as diretrizes explicitadas acima. A tabela abaixo apresenta o protocolo abrangente final, com os parâmetros escolhidos pelos pesquisadores para a realização da busca.

Quadro 2 - Critérios usados na busca da RSL.

\begin{tabular}{|c|c|}
\hline BANCOS DE DADOS & $\begin{array}{l}\text { Revistas nacionais Qualis A2 na Área de Administração, } \\
\text { RESI, JISTEN e GESTÃO.ORG e nas plataformas Spell, } \\
\text { Google Acadêmico e Portal Periódico CAPES. }\end{array}$ \\
\hline CORTE TEMPORAL & Foram buscados artigos publicados nos últimos 10 (dez) anos. \\
\hline PALAVRAS-CHAVES EM PORTUGUÊS & "modelo utaut" + "organizações públicas" \\
\hline PALAVRAS-CHAVES EM LÍNGUA ESTRANGEIRA & "utaut model" + "public organization" \\
\hline FORMA DE BUSCA & Motor de busca dos sítios eletrônicos \\
\hline CRITÉRIOS DE INCLUSÃO & $\begin{array}{l}\text { A partir da leitura do título e do resumo, identificar publicações } \\
\text { que utilizam o modelo UTAUT para compreender a adoção } \\
\text { de tecnologia da informação em organizações públicas. }\end{array}$ \\
\hline CRITÉRIOS DE EXCLUSÃO & $\begin{array}{l}\text { Foram excluídos os artigos que não abordavam o contexto } \\
\text { público; que não utilizaram o modelo Utaut; duplicados; e } \\
\text { aqueles que não foram publicados. }\end{array}$ \\
\hline AVALIAÇÃO DE QUALIDADE & Foi analisado o rigor metodológico da pesquisa. \\
\hline EXTRAÇÃO DE DADOS & Planilha simples Excel \\
\hline
\end{tabular}




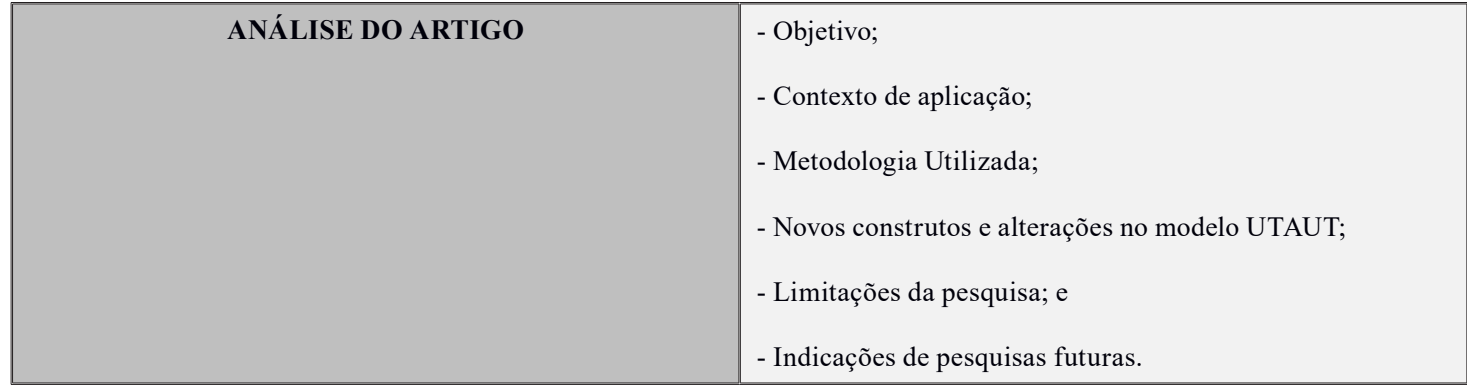

Fonte: Elaborada pelos autores.

Em relação ao banco de dados, optou-se por realizar a busca nas Revistas nacionais Qualis A2 na Área de Administração (RAC, RAE, RAUSP, BAR, CADERNOS EBAPE. BR, ORGANIZAÇÕES \& SOCIEDADE, PESQUISA OPERACIONAL, RBGN, REVISTA CONTABILIDADE \& FINANÇAS e RAP), nas revistas RESI, JISTEN e GESTÃO.ORG e nas plataformas Spell, Google Acadêmico e Portal Periódico CAPES.

No que tange ao corte temporal, definiu-se o prazo de dez anos, tendo em vista que o tema em estudo é debatido desde 2003, e, portanto, artigos mais antigos poderiam trazer contribuições relevantes para a pesquisa.

As buscas foram realizadas com as combinações de palavras-chave "modelo UTAUT" + "organizações públicas" (português) e "UTAUT model" + "public organization" (inglês). Em todos os bancos de dados a pesquisa se deu através do motor de busca.

Foi eleito o seguinte critério de inclusão: a partir da leitura do título e do resumo, identificar publicações que utilizam o modelo utaut para compreender a adoção de tecnologia da informação em organizações públicas. Consequentemente, excluíram-se os artigos que não abordavam o contexto público e aqueles que não utilizaram o modelo UTAUT. Além disso, foram excluídos também artigos duplicados e aqueles que não foram publicados.

Quanto ao critério "avaliação de qualidade", nesta pesquisa os autores observaram o rigor metodológico dos artigos, selecionando apenas aqueles que apresentaram metodologia adequada para o estudo.

Após a observância de todos os requisitos acima descritos, restaram ao total 10 (dez) artigos, os quais serão o objeto principal deste estudo. Os artigos eleitos serão analisados sob os seguintes pontos: objetivo, contexto de aplicação, metodologia, novos construtos e alterações no modelo UTAUT, limitações da pesquisa e indicação de pesquisas futuras. Tais informações foram coletadas pelos pesquisadores e organizadas em forma de planilha simples. A análise e discussão dos dados coletados será demonstrada na seção seguinte.

\section{Apresentação e análise dos resultados.}

Nesta seção, buscou-se, primeiramente, delinear algumas informações sobre as publicações encontradas nos bancos de dados, bem como realizar a análise específica dos pontos descritos no protocolo abrangente: objetivo, contexto de aplicação, metodologia, novos construtos e alterações no modelo UTAUT, limitações da pesquisa e indicação de pesquisas futuras. 


\subsection{A aplicação do modelo UTAUT em pesquisas sobre a adoção de tecnologia em organizações públicas.}

Ao realizar a busca nos bancos de dados, descritos na seção anterior, foram localizados 207 resultados, sendo 13 na Revista de Administração de Empresas (RAE); 01 na Revista de Administração da Universidade de São Paulo (RAUSP); 02 na Revista Eletrônica e Sistemas de Informação (RESI); 03 na Journal of Information Systems and Technology Management (JISTEM); 01 na GESTAO.ORG; 07 na SPELL; e 180 no GOOGLE ACADÊMICO. Nessa primeira busca, a maioria dos artigos relatava adoção de tecnologia em organizações privadas.

Após essa etapa, foram aplicados os critérios de inclusão e exclusão, realizada a avaliação de qualidade, restando ao final um total de 10 artigos, os quais foram o objeto de análise. Na tabela abaixo, estão discriminados os artigos analisados. Observa-se que a maior concentração de pesquisas sobre a temática se deu entre o período de 2014 a 2017.

Quadro 3 - Sumário dos resultados sobre os artigos aprofundados na RSL.

\begin{tabular}{|c|c|c|c|c|}
\hline 2011 & 2014 & 2014 & 2015 & 2016 \\
\hline $\begin{array}{l}\text { título: Utilização da } \\
\text { análise fatorial para } \\
\text { identificação dos fatores } \\
\text { determinantes da } \\
\text { aceitação do uso } \\
\text { de tecnologias de } \\
\text { informação na educação } \\
\text { a distância }\end{array}$ & $\begin{array}{l}\text { título: Intenção e } \\
\text { Uso de Tecnologias } \\
\text { de Informação e e } \\
\text { Comunicação } \text { pelos } \\
\text { docentes do curso } \\
\text { de Administração e } \\
\text { Ciências } \\
\text { das Contábeis } \\
\text { integrantes do sistema } \\
\text { acafe. }\end{array}$ & $\begin{array}{l}\text { autores: } \\
\text { C A S T R O ; } \\
\text { CAPPELLOZZA) } \\
\text { título: Adoção e Uso de } \\
\text { portais de governo } \\
\text { eletrônico no ambiente } \\
\text { do sistema } \\
\text { nacional de Ciência e } \\
\text { Tecnologia e } \\
\text { Inovação - } \\
\text { Um estudo baseado no } \\
\text { modelo Utaut. }\end{array}$ & $\begin{array}{l}\text { autores: MOSWEU; } \\
\text { BWALYA; MUTSHEWA) } \\
\text { título: Examining factors } \\
\text { affecting the adoption } \\
\text { and usage of document } \\
\text { workflow management } \\
\text { system (DWMS) using the } \\
\text { UTAUT model }\end{array}$ & $\begin{array}{l}\text { autores: LIMA; et al) } \\
\\
\text { título: Aplicação da } \\
\text { Teoria UTAUT no } \\
\text { Processo de Implantação } \\
\text { de um Sistema de } \\
\text { Informação para } \\
\text { Assistência Estudantil. }\end{array}$ \\
\hline 2016 & 2017 & 2017 & 2018 & 2019 \\
\hline $\begin{array}{l}\text { título: } \\
\begin{array}{l}\text { O uso de recursos } \\
\text { tecnológicos pelos } \\
\text { docentes de pós- } \\
\text { graduação } \\
\text { contabilidade: um em }\end{array} \\
\text { estudo qualitativo. }\end{array}$ & $\begin{array}{l}\text { a u t o r e s : S I L V A ; } \\
\text { WATANABE) } \\
\text { título: Aplicação } \\
\text { do Modelo Utaut na } \\
\text { Universidade Federal de } \\
\text { Rondônia: Um estudo } \\
\text { sobre a Aceitação e } \\
\text { Utilização de Sistema de } \\
\text { Informação de Gestão } \\
\text { Acadêmica. }\end{array}$ & $\begin{array}{l}\text { título: Efeito da } \\
\text { gamificação na intenção } \\
\text { de uso de treinamentos } \\
\text { on-line: Uma adaptação } \\
\text { do modelo Utaut } \\
\text { aplicado no Trt-2 }\end{array}$ & $\begin{array}{l}\text { título: Aceitação e uso } \\
\text { do sistema integrado de } \\
\text { patrimônio, administração } \\
\text { e contratos (Sipac). }\end{array}$ & $\begin{array}{l}\text { título:Implantação do } \\
\text { sistema eletrônico de } \\
\text { informações em uma } \\
\text { universidade federal da } \\
\text { Amazônia Ocidental: } \\
\text { Sob a visão da teoria } \\
\text { unificada da aceitação e } \\
\text { uso de tecnologia. }\end{array}$ \\
\hline
\end{tabular}

Fonte: Elaborado pelos autores.

Em relação ao aspecto objetivo, conforme já esperado, todos os trabalhos desenvolveram suas pesquisas no intuito de analisar a aceitação e uso de uma tecnologia ou de um sistema de informação sob a perspectiva do modelo UTAUT, porém 7 (LIMA et al, CAVALCANTE; CEOLIN; BARROS, SILVA; WATANABE, BATISTA et al., NGANGA; LEAL; FERREIRA, OLIVEIRA et al, e LEAL, et al.) especificaram já nos seus objetivos gerais essa intenção. 
Sobre o contexto da aplicação, salienta-se que, apesar do foco desta revisão sistemática ter sido selecionar apenas estudos realizados no âmbito do setor público, buscou-se investigar se dentre esses artigos existe concentração de pesquisas em determinada área, como por exemplo: educação, segurança, saúde.

Diante disso, observa-se uma concentração de estudos na área de educação, tendo em vista que, dos 10 artigos analisados, 7 foram realizados em instituições de ensino superior (BATISTA et al., LIMA et al, CAVALCANTE; CEOLIN; BARROS, SILVA; WATANABE, BATISTA et al., NGANGA; LEAL; FERREIRA, e LEAL, et al.).

Por outro lado, Costa, Castro e Cappellozza (2014) analisaram a aceitação e uso de uma tecnologia no âmbito no Ministério da Ciência, Tecnologia e Inovação; Mosweu, Bwalya e Mutshewa (2015) realizaram o estudo no Ministério do Comércio e Indústria de Botswana e Oliveira et al (2017), no Tribunal Regional do Trabalho.

No que tange à metodologia utilizada, a maioria dos autores definiu suas pesquisas como de natureza quantitativa, e 9 utilizaram questionários como instrumentos de coleta de dados. Sobre isso, destaca-se apenas os estudos de Nganga, Leal e Ferreira (2016), que definiram sua análise como uma abordagem qualitativa, optando pela realização de entrevistas como meio de levantamento de dados; e de Leal et al (2011), que utilizou métodos mistos, empregando coleta de dados associada às duas formas, qualitativa e quantitativa.

Em relação ao ponto novos construtos e alterações no modelo UTAUT, buscou-se perceber de que forma as pesquisas selecionadas aplicaram o modelo desenvolvido por Venkatesh et al (2003) no caso concreto, haja vista que o modelo UTAUT foi desenvolvido baseado em organizações do setor privado. Diante disso, o presente estudo levanta a hipótese de que ocorrem adaptações quando o modelo é aplicado no setor público.

No estudo de Lima et al (2016), verificou-se a inclusão de dois moderadores: curso e local, porém, sem apresentação de fundamentação específica sobre essa alteração no modelo. Além disso, percebe-se a ausência do moderador "voluntariedade", também sem justificativa.

Silva e Watanabe (2017), Batista et al. (2019) e Ramos et al. (2014), assim como Lima et al (2016), também não utilizaram o moderador voluntariedade, sob a justificativa de que os sistemas e/ou tecnologias analisadas são de uso obrigatório por parte dos usuários. Mosweu, Bwalya e Mutshewa (2015), por sua vez, excluíram o moderador experiência, justificando que a pesquisa possui um caráter transversal, enquanto que o estudo que originou o UTAUT ter sido longitudinal.

Cavalcante, Ceolin e Barros (2018) adicionaram aos moderadores apresentados no UTAUT o fator escolaridade, tendo em vista que a organização estudada por eles é uma Instituição de ensino superior. De acordo com os autores, a hipótese é de que os servidores com maior instrução têm uma tendência maior de aceitar o sistema objeto da pesquisa.

Sobre às adaptações realizadas nos construtos do modelo UTAUT, a análise revela alterações relevantes, como por exemplo a inclusão do construto "familiaridade" feita por Oliveira et al (2017). O estudo desenvolvido pelos referidos autores analisou a intenção de uso de treinamentos on-line realizados no Tribunal Regional do Trabalho, tendo como propósito observar características pessoais dos usuários dentro do processo de interação com a tecnologia, ou seja, 
a hipótese era a de que o usuário buscaria ambientes nos quais tivesse mais familiaridade.

Da mesma forma, Leal et al (2011) adicionaram o construto interatividade, tendo em vista o objeto de estudo ser a análise diante da Aceitação do uso de TI em Ambientes Virtuais de Aprendizagem na educação a distância. Com essa inclusão, os autores avaliaram a percepção de interatividade entre tutor e alunos. Já na pesquisa de Nganga, Leal e Ferreira (2016), foi inserido o construto "concepção pedagógica", o qual, de acordo com os autores, refere-se às crenças que o docente tem sobre o processo ensino-aprendizagem.

Por fim, apenas o estudo de Costa, Castro e Cappellozza (2014) utilizou a aplicação clássica proposta por Venkatesh et al (2003), ou seja, sem alteração.

Nesse sentido, pode-se afirmar que essas investigações apresentadas sugerem adaptações no modelo UTAUT, porém, não fica claro se essas alterações foram motivadas pelas características da tecnologia adotada nas pesquisas ou pelo contexto do serviço público.

A respeito das limitações apresentadas pelos autores, percebe-se a predominância do aspecto metodológico, no tocante à composição da amostra, tendo em vista a aplicação majoritária de questionário como instrumento de coleta de dados (adaptado do estudo de Venkatesh et al., (2003)). Assim, os resultados identificados não puderam ser generalizados.

Destaca-se a limitação apresentada por Costa, Castro e Cappellozza (2014), apresentada nos seguintes termos: “[...] entre outras limitações do estudo, considera-se que o resultado dos valores obtidos nas análises pode ser diferente em razão das características de outras tecnologias, além de aspectos individuais e outros contextos de uso dos recursos."

Sobre as disfunções que podem ser encontradas nos estudos sobre o modelo UTAUT, faz-se necessário trazer ao debate considerações de Brito e Ramos (2019) referentes ao estudo Limitações dos Modelos de Aceitação da Tecnologia: um ensaio sob uma perspectiva crítica, no qual foi realizada uma exposição crítica sobre limitações dos principais modelos e teorias de aceitação de tecnologia existentes, entre elas o UTAUT:

[...] destaca-se que a UTAUT se apresenta como um modelo de aceitação da tecnologia com alta capacidade explicativa. Porém também não se pode dizer que se trata de um modelo ideal, tendo em vista que não há um consenso quanto aos níveis de resposta das suas variáveis (Rahman, et al., 2017). Assim sendo, pondera-se que mesmo esse modelo, com um maior número de variáveis, não consegue responder de forma contundente todos os fatores que exercem influência sobre o indivíduo na tomada de decisão quanto ao uso (ou não) de uma tecnologia da informação. (Brito e Ramos, 2019, p.216)

Contudo, ainda sobre essa questão, Brito e Ramos (2019) concluem que o modelo UTAUT é eficaz no que se propõe e que as limitações existentes são decorrentes da complexidade do fenômeno que ele busca tratar, "sendo pouco provável a existência de um modelo ótimo capaz de responder de forma completa a questões sobre quais os fatores que influenciam o uso de uma tecnologia da informação" (BRITO; RAMOS, 2019). Diante disso, cabe salientar que esta pesquisa entende que esses fatores limitadores não restringem a relevância das pesquisas sobre a temática.

Por fim, no que concerne às pesquisas futuras, os autores apresentaram sugestões, dentre as quais, destacam-se: ampliação do estudo sobre aceitação e uso de um sistema em di- 
ferentes grupos para avaliar eventuais diferenças (COSTA, CASTRO, CAPPELLOZZA, 2014); ampliação de moderadores com aspectos motivacionais como sobrecarga de atividades (SILVA; WATANABE, 2017); investigar fatores descobertos nas entrevistas como computer anxiety, incompatibilidade de DWMS com as práticas de trabalho, além de atitudes negativas em relação ao uso do sistema e complexidade do sistema (MOSWEU; BWALYA; MUTSHEWA, 2015).

\section{Considerações finais.}

Através desta revisão sistemática da literatura, analisou-se sistematicamente a literatura que utiliza o modelo UTAUT para compreender a adoção de tecnologia em organizações públicas, a partir dos seguintes aspectos de análise: objetivo dos artigos encontrados; contextos de aplicação; metodologia utilizada; construtos adaptados, limitações da pesquisa e indicações de pesquisas futuras. Ademais, como contribuição complementar, o artigo buscou construir um protocolo de revisão sistemática abrangente.

O número de artigos presentes na primeira busca demonstra que o contexto público tem sido preterido pelos pesquisadores, quando comparado às pesquisas que estudam adoção de tecnologia em organizações privadas. Os resultados indicam que há lacunas a serem investigadas. Menciona-se, como exemplo, o fato de que a maioria das investigações dos artigos selecionados sofreram adaptações no modelo UTAUT, porém, não fica claro, somente com os achados deste estudo, se essas alterações foram motivadas pelas características da tecnologia adotada ou pelo contexto do serviço público. Além disso, a análise dos pontos "limitações" e "pesquisas futuras", nos artigos selecionados, demonstram que o tema ainda não foi esgotado.

$\mathrm{O}$ artigo ainda apresenta uma proposta de protocolo abrangente, elaborado a partir de revisões sistemáticas selecionadas em etapa anterior ao objetivo principal. Essas revisões permitiram construir um protocolo com os seguintes elementos: banco de dados; corte temporal; palavras-chave em português; palavras-chave em língua estrangeira; forma de busca; critérios de inclusão; critérios de exclusão; avaliação de qualidade dos artigos; extração de dados, e, por fim, a análise dos artigos.

A respeito das limitações enfrentadas durante a revisão sistemática, destaca-se o número reduzido de publicações que atendessem a todos os critérios de inclusão eleitos, principalmente a aplicação do modelo UTAUT no contexto do serviço público. Já em relação às pesquisas futuras, sugere-se a ampliação de estudos sobre a aplicação do modelo UTAUT em diversos contextos públicos, tendo em vista que os resultados apontam uma prevalência de estudos na área de educação. Ademais, deve ser investigado se a necessidade de adaptação do modelo advém do tipo de tecnologia empregada na organização ou do próprio contexto público, de modo a responder a lacuna identificada pelos pesquisadores. Certamente, essa ampliação será pertinente ao avanço da literatura sobre Aceitação e Uso de Tecnologia nas organizações públicas. 


\section{Referências}

ANDWIKA, V. R. .; WITJAKSONO, R. W. . Analysis of User Acceptance of ERP System on After Sales Function Using Unified Theory of Acceptance and Use of Technology (UTAUT) Model. International Journal of Advances in Data and Information Systems, v. 1, n. 1, p. 26-33, 1 Apr. 2020.

ARAÚJO, J. I. S. et. al. Benefícios e limitações da simplicidade em Projetos Inovadores de Software: uma revisão sistemática da literatura. Revista Gestão. Org., v. 16, p. 279-292, 2018.

BATISTA, Rosana Cláudia Smék et al. IMPLANTAÇÃO DO SISTEMA ELETRÔNICO DE INFORMAÇÕES EM UMA UNIVERSIDADE FEDERAL DA AMAZÔNIA OCIDENTAL: SOB A VISÃO DA TEORIA UNIFICADA DE ACEITAÇÃO E USO DA TECNOLOGIA.. In: XIX Colóquio Internacional de Gestão Universitária, Florianópolis-SC. 2019.

BRESSER PEREIRA, Luiz Carlos. A reforma do Estado dos anos 90: lógica e mecanismos de controle. Lua Nova: Revista de cultura e política, n. 45, p. 49-95, 1998.

BRITO, J. V. da C. S; RAMOS, A. S. M. Limitações dos modelos de Aceitação da Tecnologia: Um ensaio sob uma perspectiva crítica. Revista Gestao.Org, v.17, edição especial, 2019.

CASSUNDÉ, F.; BARBOSA, M.; MENDONÇA, J. Entre revisões sistemáticas e bibliométricas: como tem sido mapeada a produção acadêmica em administração no Brasil? Inf. Inf, Londrina, v. 23, n. 1, p. 311-334, 2018.

CAVAlCANTE, G. M. ; CEOLIN, A. C. ; BARROS, J. E. M. . Aceitação e Uso do Sistema de Patrimônio, Administração e Contratos (SIPAC).. RGO. REVISTA GESTÃO ORGANIZACIONAL (ONLINE), v. 11, p. 79-96-96, 2018.

COSTA, Érico da Silva ; CASTRO, D. S. P. ; CAPPELlOzZA, A. . ADOÇÃO E USO DE PORTAIS DE GOVERNO ELETRÔNICO NO AMBIENTE DO SISTEMA NACIONAL DE CIÊNCIA E TECNOLOGIA E INOVAÇÃO: UM ESTUDO BASEADO NO MODELO UTAUT. RECADM : Revista Eletrônica de Ciência Administrativa , v. 13, p. 286-300, 2014.

COUTINHO, M. J. V. Administração pública voltada para o cidadão: quadro teórico-conceitual. Revista do Serviço Público, v. 51, n. 3, p. 40-73, 2014.

FERREIRA, J. E.; PINTO, F. G. C.; SANTOS, S. C. Estudo de mapeamento sistemático sobre as tendências e desafios do Blockchain. Revista Gestão.Org, v. 15, p. 108-117, 2017.

FONSECA, P. G. et al. Critical success factors of erp implementation in public organizations: scientific production on brazilian events. In: 14th CONTECSI-International Conference on Information Systems and Technology Management. 2017a.

FONSECA, P. G. Influência do gerenciamento dos elementos dos fatores críticos de sucesso de Enterprise Resource Planning no setor público sob a ótica de pressões institucionais / Platini 
Gomes Fonseca. Tese. UFBA - Salvador - 2019.

FONSECA, P. G.; SANTOS, E. M.; ALBUQUERQUE JUNIOR, A. E. Oito X Oito: uma revisão sistemática da literatura sobre os Fatores Críticos de Sucesso para implementação de ERP - enfatizando as organizações públicas. In: SEMINÁRIOS DE ADMINISTRAÇÃO, 20., 2017. São Paulo. Anais... São Paulo: FEA/USP, 2017 b.

FONSECA, P. G; SANTOS, E. M; ALBUQUERQUE JUNIOR, A; DALTRO, E. F. Critical success factors for erp implementation in public organizations: a gap in specialized bibliography? 15th International conference on information systems \& technology management, 2018.

GONZALES et al. Teoria Unificada de Aceitação e Uso da Tecnologia: Revisão do UTAUT como Estrutura Conceitual em Eventos Científicos Brasileiros. In: 17. Conferência da Associação

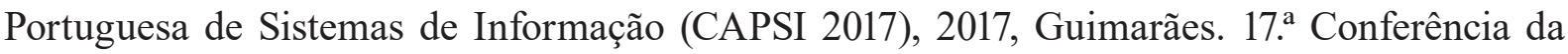
Associação Portuguesa de Sistemas de Informação, 2017.

GONZALEZ JUNIOR, Ivo Pedro. Adoção e infusão de Ambientes Virtuais de Aprendizagem (AVA) para suporte ao ensino presencial; 2017; Tese (Doutorado em Administração) Universidade Federal da Bahia,

GOVERNO FEDERAL. Gov.br, 2020. Do Eletrônico ao Digital: Linha do tempo. Disponível em: $\quad$ https://www.gov.br/governodigital/pt-br/estrategia-de-governanca-digital/doeletronico-a0-digital $>$. Acesso em: 24, agosto e 2020.

GUIMARAES, T.A; MEDEIROS, P. H. R. A relação entre governo eletrônico e governança eletrônica no governo federal brasileiro. Cad. EBAPE.BR [online]. 2005, vol.3, n.4, p. 01-18

GUIMARAES, T.A; MEDEIROS, P. H. R. A relação entre governo eletrônico e governança eletrônica no governo federal brasileiro. Cad. EBAPE.BR [online]. 2005, vol.3, n.4, p. 01-18.

KITCHENHAM, B.; CHARTERS, S. Guidelines for Performing Systematic Literature Reviews in Software Engineering. Keele University, 2007.

LAUDON, K. C.; LAUDON, J. P. Sistemas de informação gerenciais. 11. ed. São Paulo: Pearson, 2014. p.34.

LEAL, Edvalda Araújo. Utilização da Análise Fatorial para Identificação dos Fatores Determinantes da Aceitação do Uso de Tecnologias de Informação na Educação a Distância. 2011. In: XXXV EnANPAD (Congresso),Rio de Janeiro- RJ,2011.

LIMA, T. P. ; BENEVIDES, S. L. M. ; WATANABE, C. Y. V. ; SILVA, R. M. P. ; RODRIGUES, T. D. M. . Aplicação da Teoria UTAUT no Processo de Implantação de um Sistema de Informação para Assistência Estudantil. In: I Congresso Nacional PROFIAP, 2016, Curitiba. Políticas Públicas: Formulação e Gestão, 2016. v. 4.

LOPES, K. M. G.; LUCIANO, E. M.; MACADAR, M. A. Criando valor público em serviços 
digitais: uma proposta de conceito. Revista Gestão.Org, v. 16, p. 207-221, 2018.

MAGNAGNAGNO, O. A.; LUCIANO, E. M.; WIEDENHÖFT, G. C. Redução dos Níveis de Corrupção no Brasil: qual o papel da tecnologia da informação e comunicação? Revista Gestão.Org, v. 15, p. 157-170, 2017.

MIRANDA, A. C. C.; VERÍSSIMO, A. M.; CEOLIN; A. C. Agricultura de precisão: um mapeamento da base da Scielo. Revista Gestão.Org, v. 15, p. 129-137, 2017.

MIRANDA, W. F. ; RICCIO, E. L. . Antecedentes da aceitação e adoção da auditoria contínua no setor público brasileiro: O caso do Tribunal de Contas do Estado de São Paulo. In: XVIII USP International Conference in Accounting, 2018.

MOSWEU, Olefhile; BWALYA, Kelvin; MUTSHEWA, Athulang. Examining factors affecting the adoption and usage of Document Workflow Management System (DWMS) using the UTAUT model. Records Management Journal, 2016.

NASCIMENTO, S. G. V.; FREIRE, G. H. A.; DIAS, G. A.. A tecnologia da informação e a gestão pública. Gestão \& Aprendizagem, v. 1, n. 1, p. 167- 182, 2012.

NEVES, M. P. B. et al. Os Desafios no Uso de Tecnologias na Governança Eletrônica: o que diz a produção acadêmica nacional? Revista Gestao.Org, v.17, 2019.

NGANGA, C. S. N. ; LEAL, E. A. ; FERREIRA, L. V. . O uso de recursos tecnológicos pelos docentes de pós-graduação em contabilidade: um estudo qualitativo. In: XVI Congresso USP Controladoria e Contabilidade, 2016, São Paulo. Anais do XVI Congresso USP Controladoria e Contabilidade, 2016.

OLIVEIRA, ITALO MARTINS DE. Usabilidade do Sistema Integrado de Patrimônio, Administração e Contratos (SIPAC) pela Universidade Federal da Paraíba (UFPB). Tese de mestrado. João Pessoa, 2017, pp 33- 38.

OLIVEIRA, Ludmilla Cavarzere de ; PINOCHET, L. H. C. ; BUENO, R. L. P. ; OLIVEIRA, M. A. . EFEITO DA GAMIFICAÇÃO NA INTENÇÃO DE USO DE TREINAMENTOS ON-LINE: UMA ADAPTAÇÃO DO MODELO UTAUT APLICADO NO TRT-2. Revista de administração da UFSM , v. 12, p. 472-491, 2019.

RAMOS, S. P. ; MONDINI, Vanessa Edy Dagnoni ; DOMINGUES, Maria José Carvalho de Souza ; SOETHE, J. S. . Intenção e uso de tecnologias de informação e comunicação pelos docentes do curso de administração e Ciências Contábeis das universidades integrantes do sistema ACAFE. In: Colóquio Internacional de Gestão Universitária, 2014, Florianópolis. XIV Colóquio Internacional de Gestão Universitária, 2014.

RORATTO, R.; DIAS, E. D. Fatores de risco no gerenciamento de projetos de tecnologia da informação em instituições públicas. Revista de Economia e Administração, v.11, n.3, p. 386399, jul./set. 2012. 
ROZTOCKI, N; STRZELCZYK, W. Enterprise Systems in the Public Sector: A Literature Review. AMCIS 2020 Proceedings. 23, 2020.

SANTOS, A. F. C.; DEDA, R. R.; OLIVEIRA, A. A. Identificação de ontologias com BPM no ambiente da saúde: Uma revisão sistemática. Revista Gestão.Org, v. 13, p. 176-186, 2015.

SENA, A. S; GUARNIERI, P. Enterprise Resource Planning governamental: a percepção dos servidores atuantes no Projeto Ciclo do Ministério da Justiça quanto à implementação. Revista de Administração Pública, v. 49, n. 1, p. 207-230, 2015.

SILVA, A. P. ; Watanabe, Carolina Y. V. . Aplicação do modelo UTAUT na Universidade Federal de Rondônia: Um estudo sobre a aceitação e utilização de sistema de informação de gestão acadêmica. REVISTA ELETRÔNICA DE SISTEMAS DE INFORMAÇÃO (RESI), v. 16, p. 1-23, 2017.

SILVA, C. J. S.; GARCIA, V. C.; NASCIMENTO, L. M. Estudo de Mapeamento Sistemático sobre as Tendências e Desafios do Cloud Gaming. Revista Gestão.Org, v. 14, p. 224-233, 2016.

SILVA, R. F. M.; BURÉGIO, V. A. A. Um estudo de mapeamento das contribuições e desafios de pesquisa em máquinas sociais. Revista Gestão.Org, v. 16, p. 245-257, 2018.

SOLIMAN, Mohamed Soliman Mohamed et al. Modelling Intention to Use ERP Systems among Higher Education Institutions in Egypt: UTAUT Perspective. Int. J Sup. Chain. Mgt Vol, v. 8, n. 2, p. 429, 2019.

VENKATESH, V; MORRIS, M. G; DAVIS, G. B; DAVIS, F. D. User acceptance of information technology: toward a unified view. MIS Quarterly, Minneapolis, 27, 3, 425-478, 2003.

VENKATESH, Viswanath. et al. User acceptance of information technology: toward a unified view. MIS Quarterly v. 27, n. 3, September 2003, p. 425-478. 